\title{
Eficácia da fenestração da lâmina terminal associada à fenestração da membrana de Liliequist na prevenção de hidrocefalia crônica em pacientes operados na fase aguda por aneurisma cerebral roto
} Efficacy of Lamina Terminalis Fenestration Associated whith Liliequist' Membrane in reducing ShuntDependent Hydrocephalus following Aneurysmal Subarachnoid Hemorrhage

\author{
Marcelo Minamoto Miyabe ${ }^{1}$ Juan Antonio Castro Flores ${ }^{2}$ Jose Carlos Esteves Veiga ${ }^{3}$ \\ ${ }^{1}$ Residente de Neurocirurgia no Hospital Central da Santa Casa de \\ Misericórdia de São Paulo, São Paulo, SP, Brasil \\ 2 Assistente da Disciplina de Neurocirurgia do Hospital Central da \\ Santa Casa de Misericórdia de São Paulo, São Paulo, SP, Brasil \\ ${ }^{3}$ Chefe da Disciplina de Neurocirurgia do Hospital Central da Santa \\ Casa de Misericórdia de São Paulo, São Paulo, SP, Brasil \\ Arq Bras Neurocir 2015;34:175-178.

\begin{abstract}
Address for correspondence Juan Antonio Castro-Flores, MR, Rua CEP: 04116-020 (e-mail: castroja@me.com).
\end{abstract} \\ Prof. Carolina Ribeiro, 30, ap. 91, Vila Mariana, São Paulo, SP, Brasil
}

\section{Resumo \\ Palavras-Chave \\ - hidrocefalia \\ - hemorragia subaracnóidea \\ - aneurisma \\ - fenestração \\ - lâmina terminal \\ - membrana de Liliequist}

Objetivos Hidrocefalia tardia que requer implante de derivação liquórica é frequente após hemorragia subaracnóidea por aneurisma cerebral roto, e contribui para a morbimortalidade tardia. Alguns autores reportam que a fenestração microcirúrgica da Lâmina Terminal, durante a cirurgia do aneurisma diminui a incidência de hidrocefalia tardia.

Material e Método No período de Janeiro de 2010 a Janeiro de 2012 realizamos a fenestração da Lâmina Terminal associada à fenestração da Membrana de Liliequist, em 17 pacientes operados na fase aguda por aneurisma roto. Monitoramos por tomografia a presença de hidrocefalia após 6 e 16 meses.

Resultados Nenhum paciente apresentou hidrocefalia tardia.

Conclusão A fenestração da Lâmina Terminal associada à fenestração da Membrana de Liliequist é eficaz na prevenção da hidrocefalia tardia pós hemorragia subaracnóidea por aneurisma roto.

Objectives Chronic hydrocephalus requiring shunt placement is common following aneurysm subarachnoid hemorrhage, and contributes to the late morbidity and mortality Some authors report that microsurgery fenestration of Lamina Terminalis during aneurysm surgery affords a reduction in the development shunt-dependent hydrocephalus.

Methods From January 2010 to January 2012 we performed microsurgery fenestration of Lamina Terminalis and Liliequist's Membrane, in 17 patients operated in the acute phase. CT scans were performed after 6 and 16 months

Result There was no development of hydrocephalus in this series. received

July 22, 2014

accepted

June 12, 2015
DOI http://dx.doi.org/ 10.1055/s-0035-1559891. ISSN 0103-5355.
Copyright $(2015$ by Thieme Publicações License terms Ltda, Rio de Janeiro, Brazil

(c) (i) $\ominus$ (5) 
Conclusion Microsurgery fenestration of Lamina Terminalis associated with Liliequist's Membrane fenestration is effective in preventing late hydrocephalus after subarachnoid hemorrhage due to ruptured aneurysm.

\section{Introdução}

Uma complicação frequente na hemorragia subaracnóidea (HSA) por aneurisma roto é a hidrocefalia. Pode ocorrer precocemente (15-87\%) ou tardiamente (8,9-48\%). A hidrocefalia precoce requer tratamento imediato. ${ }^{1}$

A hidrocefalia crônica contribui com a morbi-mortalidade tardia na HSA. Acredita-se que seja causada por fibrose da leptomeninge e das granulações aracnoides, prejudicando a circulação e absorção de líquor. ${ }^{2} \mathrm{O}$ tratamento é a derivação ventriculoperitoneal (DVP) (nível de evidência C, classe 1). ${ }^{1}$

Um problema adicional, que contribui com a morbimortalidade tardia, é representado pelas complicações da DVP (13\%), sejam infecciosas ou mecânicas.

Alguns autores reportam que a fenestração microcirúrgica da lâmina terminal, durante a cirurgia do aneurisma, reduz a incidência de hidrocefalia tardia. ${ }^{2-4} \mathrm{~A}$ fenestração da lâmina terminal proporciona uma terceiro-ventriculostomia anterior, facilitando a dinâmica do fluxo liquórico, reduzindo a fibrose do tecido subaracnóideo e diminuindo a inflamação vascular. $^{2,3}$ Nesta linha de raciocínio, acreditamos que a fenestração da membrana de Liliequist pode ser uma medida complementar eficaz.

A membrana de Liliequist está localizada entre as cisternas interpedunculares e quiasmática. Yasargil a descreveu como "uma cortina que se estende de uma face mesial temporal à outra." Matsuno identificou duas folhas, a diencefálica, que se estende superiormente do diencéfalo posterior até os corpos mamilares e separa a cisternas quiasmáticas e interpedunculares, e a segunda, chamada de membrana mesencefálica, que se estende posteriormente se inserindo na junção ponto-mesencefálica, e separa as cisternas interpedunculares e pré-pontinas. Brasil e Schneider a descrevem como três paredes intercisternais: carotídeo-interpeduncular direita e esquerda e parede central quiasmática interpeduncular (-Fig. 4). ${ }^{5}$

Este estudo objetiva avaliar a eficácia da fenestração microcirúrgica da lâmina terminal associada à fenestração da membrana de Liliequist, na prevenção de hidrocefalia crônica, em uma série de 17 pacientes operados na fase aguda por aneurisma roto.

\section{Material e Métodos}

No período de janeiro de 2010 a janeiro de 2012 foram realizadas oitenta craniotomias para tratamento de noventa aneurismas cerebrais, no Hospital Central da Irmandade da Santa Casa de São Paulo. A mortalidade foi de 17,5\% (14 óbitos).
Seis pacientes $(7,5 \%)$ evoluíram com hidrocefalia tardia, sendo necessária a derivação ventriculoperitoneal. Em nenhum destes pacientes havia sido realizada a fenestração da lâmina terminal nem da membrana de Liliequist.

Realizamos a fenestração microcirúrgica da lâmina terminal associada à fenestração da membrana de Liliequist em 17 pacientes operados na fase aguda. A maioria apresentava hemorragia subaracnóidea importante (-Tabela $\mathbf{1}$ ).

Após a clipagem do aneurisma, foi realizada ampla fenestração da lâmina terminal, e a seguir a membrana de Liliequist foi fenestrada lateral e medialmente à carótida interna, no mesmo campo operatório, até a visualização da artéria basilar e segmento P1 da artéria cerebral posterior bilateral. Em alguns casos, foi realizada a fenestração da lâmina terminal antes da clipagem do aneurisma para obter relaxamento cerebral (-Figs. 1-3).

A mortalidade nesta série foi de 5,8\% (um paciente de 84 anos, faleceu no $18^{\circ}$ dia de pós-operatório por complicações clínicas, sem sinais de hidrocefalia na tomografia de crânio).

Foi avaliada a tomografia computadorizada de crânio tardia (6-16 meses de pós-operatório). Nenhum paciente apresentou hidrocefalia.

\section{Discussão}

A hidrocefalia é uma complicação frequente em HSA por aneurisma roto. O tratamento de hidrocefalia precoce é emergencial. A derivação ventricular ou lombar externa estão relacionadas com melhora clínica e diminuição da incidência de vasoespasmo.

Durante o tratamento para a hidrocefalia crônica e a DVP podem ocorrer complicações mecânicas ou infecciosas (13\%) que aumentam a morbi-mortalidade e elevam os custos de tratamento. É razoável investir em tratamento preventivo.

A fenestração da lâmina terminal foi proposta para tentar reduzir a incidência de hidrocefalia tardia. Uma metanálise de 11 estudos não randomizados não encontrou diferença significativa entre ocorrência de hidrocefalia tardia em pacientes submetidos a fenestração da lâmina terminal. ${ }^{2}$

Tabela 1 Grau da HSA na tomografia de crânio

\begin{tabular}{|l|l|}
\hline Fisher 1 & 2 pacientes \\
\hline Fisher 2 & 4 pacientes \\
\hline Fisher 3 & 9 pacientes \\
\hline Fisher 4 & 2 pacientes \\
\hline Total & 17 pacientes \\
\hline
\end{tabular}




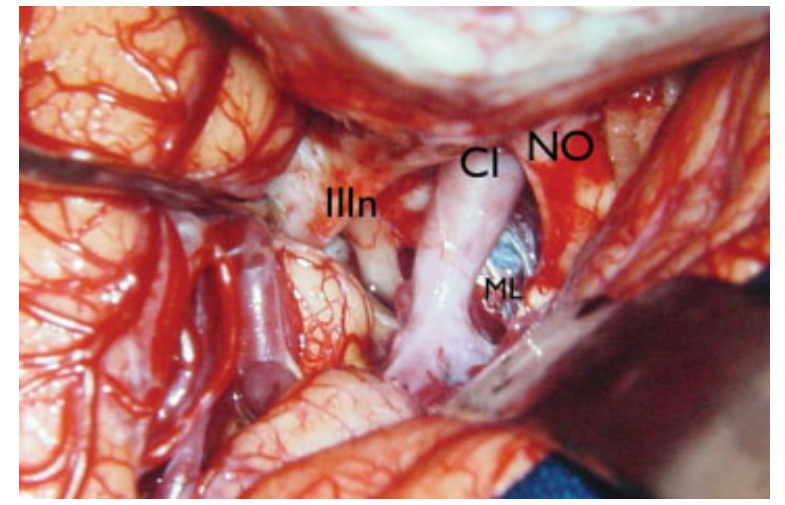

Fig. 1 Exposição da membrana de Liliequist (ML) entre a carótida interna (CI) e o nervo óptico esquerdo (NO). Lateralmente, o nervo III (IIIn).

A membrana de Liliequist constitui um ponto de referência anatômico para vários procedimentos neurocirúrgicos e participa da fisiopatogenía de alguns processos patológicos:

- tumores que ocupam as cisternas basais (craniofaringiomas, meningiomas) com frequência não comprometem a integridade anatômica da membrana de Liliequist, servindo como parâmetro do plano de dissecção;

- falha de terceiro-ventriculostomia endoscópica devido à falta de fenestração da membrana de Liliequist $^{6}$;

- cisto aracnóideo suprasselar: a membrana de Liliequist determina a classificação de dois tipos de cisto: (1) intraaracnóideo da cisterna diencefálica e (2) dilatação cística da cisterna interpeduncular (entre as duas folhas da membrana de Liliequist) ${ }^{7}$;

- hemorragia perimesencefálica: localização mais frequente nas cisternas interpeduncular e prepontina; Shwartz e Solomon sugerem que a membrana de Liliequist é capaz de circunscrever a hemorragia na cisterna interpeduncular especialmente em hemorragias venosas de baixa pressão ou de pequeno volume (-Figs. 4 e 5).$^{5}$

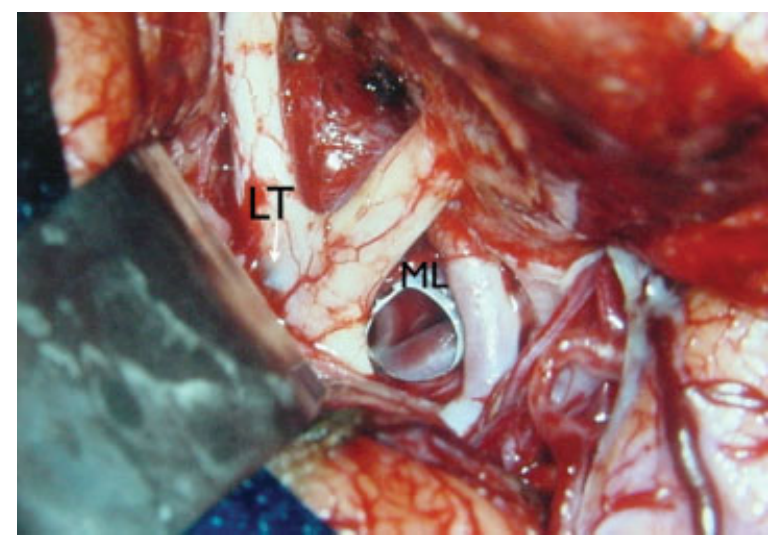

Fig. 2 Exposição da Lâmina Terminal (LT). Lateralmente se observa fenestração da Membrana de Liliequist (ML), com exposição da artéria basilar.

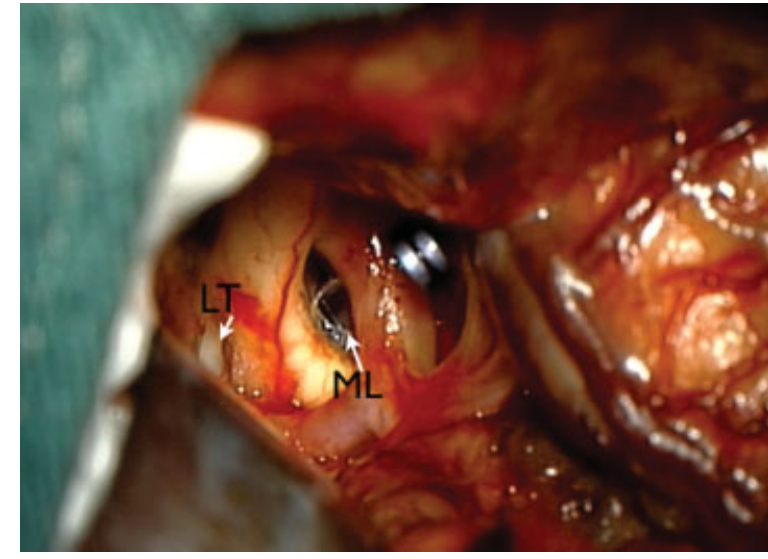

Fig. 3 Aspecto final da fenestração da lâmina terminal (LT) e da membrana de Liliequist (ML).

A membrana de Liliequist constitui uma barreira entre as cisternas basais supra e infratentoriais. A sua fenestração facilita o acesso inferolateral ao assoalho do terceiro ventrículo e proporciona uma via adicional para a circulação de líquor. Acreditamos que representa um complemento à fenestração da lâmina terminal, resultando em uma medida eficaz na prevenção da hidrocefalia tardia.

Na série apresentada nenhum paciente desenvolveu hidrocefalia. Os seis pacientes que necessitaram de DVP não haviam sido submetidos a fenestração da lâmina terminal nem da membrana de Liliequist.

Observamos que em vários pacientes, mesmo depois da dissecção das cisternas supratentoriais, há saída de líquor "represado" ao fenestrar a membrana de Liliequist. Em todos os casos, realizamos a limpeza mecânica "maximizada" dos coágulos cisternais (fator reconhecido para a prevenção de hidrocefalia e isquemia). ${ }^{4}$ Casos operados na fase tardia poderiam ter maior chance de desenvolver hidrocefalia crônica.

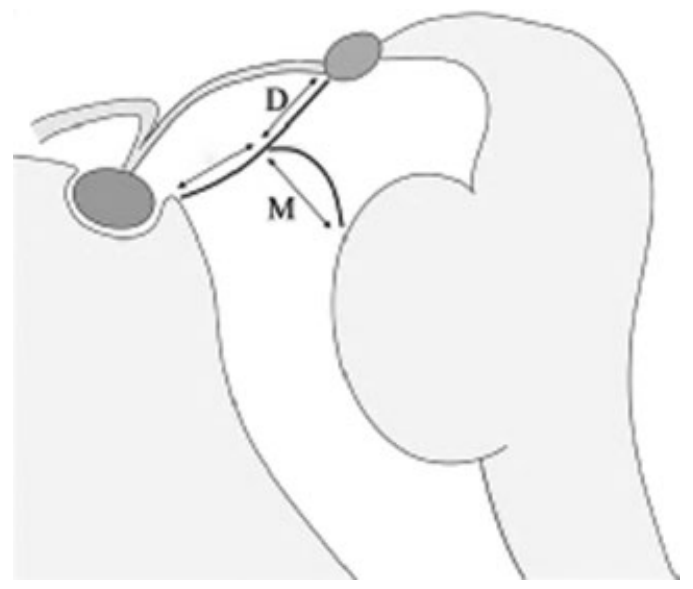

Fig. 4 Desenho esquemático das folhas diencefálica (D) e mesencefálica (M) da membrana de Liliequist. 
178 Eficácia da fenestração da lâmina terminal associada à membrana de Liliequist Miyabe et al.

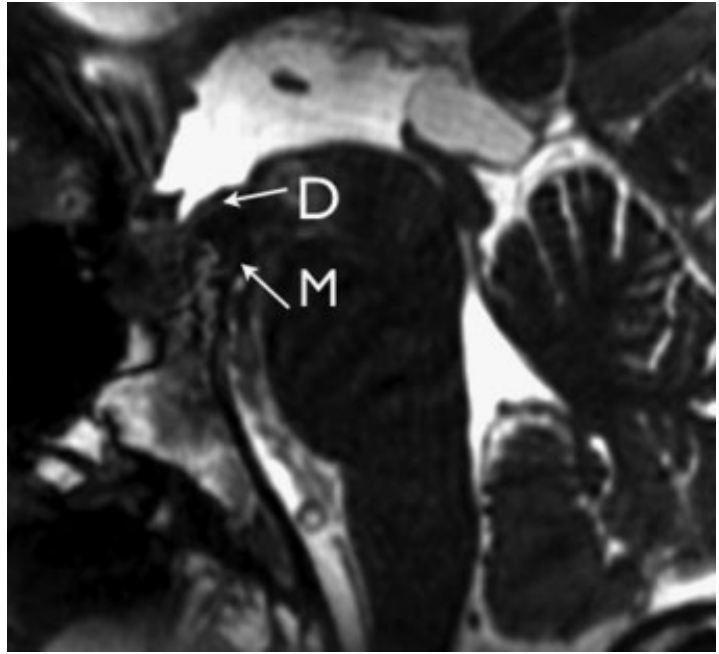

Fig. 5 Ressonância magnética, sequência FIESTA, em paciente com hemorragia perimesencefálica demonstrando abaulamento das foIhas diencefálica (D) e mesencefálica (M) circunscrevendo a hemorragia.

\section{Conclusão}

A fenestração microcirúrgica da lâmina terminal, complementada com a fenestração da membrana de Liliequist, constitui um refinamento da cirurgia precoce do aneurisma roto. Em nossa série, foi efetiva na prevenção da hidrocefalia crônica.

\section{Referências}

1 Connolly ES Jr, Rabinstein AA, Carhuapoma JR, et al; American Heart Association Stroke Council; Council on Cardiovascular Radiology and Intervention; Council on Cardiovascular Nursing; Council on Cardiovascular Surgery and Anesthesia; Council on Clinical Cardiology. Guidelines for the management of aneurysmal subarachnoid hemorrhage: a guideline for healthcare professionals from the American Heart Association/american Stroke Association. Stroke 2012;43(6):1711-1737

2 Komotar RJ, Hahn DK, Kim GH, et al. Efficacy of lamina terminalis fenestration in reducing shunt-dependent hydrocephalus following aneurysmal subarachnoid hemorrhage: a systematic review. Clinical article. J Neurosurg 2009;111(1):147-154

3 Komotar RJ, Hahn DK, Kim GH, et al. The impact of microsurgical fenestration of the lamina terminalis on shunt-dependent hydrocephalus and vasospasm after aneurysmal subarachnoid hemorrhage. Neurosurgery 2008;62(1):123-132, discussion 132-134

4 Mura J, Rojas-Zalazar D, Ruíz A, Vintimilla LC, Marengo JJ. Improved outcome in high-grade aneurysmal subarachnoid hemorrhage by enhancement of endogenous clearance of cisternal blood clots: a prospective study that demonstrates the role of lamina terminalis fenestration combined with modern microsurgical cisternal blood evacuation. Minim Invasive Neurosurg 2007;50(6):355-362

5 Froelich SC, Abdel Aziz KM, Cohen PD, van Loveren HR, Keller JT. Microsurgical and endoscopic anatomy of Liliequist's membrane: a complex and variable structure of the basal cisterns. Neurosurgery 2008;63(1, Suppl 1):ONS1-ONS8, discussion ONS8-ONS9

6 Buxton N, Vloeberghs M, Punt J. Liliequist's membrane in minimally invasive endoscopic neurosurgery. Clin Anat 1998;11(3):187-190

7 Miyajima M, Arai H, Okuda O, Hishii M, Nakanishi H, Sato K. Possible origin of suprasellar arachnoid cysts: neuroimaging and neurosurgical observations in nine cases. J Neurosurg 2000;93(1):62-67 